\title{
Glass Transition of Oligostyrene with Different End Groups
}

\author{
Tatsuko Hatakeyama and Mitsuru Serizawa* \\ Research Institute for Polymers and Textiles, \\ 1-1-4, Yatabe-Higashi, Tsukuba, Ibaraki 305, Japan.
}

(Received July 15, 1981)

\begin{abstract}
Oligostyrenes having butyl groups at both ends, a butyl group at one and a proton at the other, and protons at both ends were prepared. The oligomers obtained were separated into fractions from monomer to octamer by the combined use of vacuum distillation and gel permeation chromatography (GPC). The purity of each fraction was characterized by GPC. The monomer and dimer having butyl groups at both ends were found to crystallize; melting and premelt crystallization were detected by differential scanning calorimeter (DSC). All the oligomer fractions and the monomer became glassy when quenched from the molten state. The glass transition temperatures $\left(T_{\mathrm{g}}\right.$ 's) of the oligomers having the same number of main chain segments were similar in spite of the difference in molecular weight $\left(M_{n}\right)$. Thus, the values of the constants in the equation of $T_{\mathrm{g}}=A-B / M_{n}$ (where $A$ and $B$ are constants) varied depending on the type of sample in the range of molecular weight up to 600 . This fact indicates that the molecular motion of the end groups cancels out the effect of increasing molecular weight.

KEY WORDS Oligostyrene / End Group / Fractionation / Molecular Weight / Glass Transition / Differential Scanning Calorimetry /
\end{abstract}

The molecular weight at which polymolecularity appears in polymers has received much attention. The molecular weight dependence of physicochemical properties of polymers has been discussed in a large number of reports. In particular, the variation in glass transition temperature $T_{\mathrm{g}}$ with the molecular chain length is well established for various kinds of amorphous polymers. ${ }^{1-7}$ The value of $T_{\mathrm{g}}$ for a given polymer varies remarkably in the low molecular weight region and attains a characteristic temperature at a certain molecular weight. Oligometric materials are considered to be a suitable model for studying the variation of $T_{\mathrm{g}}$ and also the appearance of the polymolecularity. In spite of the importance of oligomers, few papers have been concerned with the relation between $T_{\mathrm{g}}$ and chain length in the low molecular weight region. ${ }^{8,9}$ Uebbereiter and $\mathrm{Kanig}^{8}$ synthesized styrene oligomers by bulk polymerization and separated them into different molecular weight fractions. They observed $T_{\mathrm{g}}$ by dilatometry and explained the observed relationship between $T_{\mathrm{g}}$ and molecular weight

* Present address: Organon Japan, 1-13-13, Ginza, Chuo-ku, Tokyo 104, Japan. by introducing the self-plasticization effect of the end groups.

Recently, Cowie ${ }^{10}$ systematically studied the transition temperature of various kinds of oligomers and replotted all the available data for $T_{\mathrm{g}}$ against the molecular weight. He found a characteristic molecular weight at which $T_{\mathrm{g}}$ varied discontinuously.

In these studies, oligomer purity is not specified, but $T_{\mathrm{g}}$ is known to be affected by molecular weight distribution. Samples having narrow molecular weight distribution exhibit high $T_{\mathrm{g}}$, even in the high molecular weight region.

In this study, we prepared two kinds of fractionated styrene oligomers having different end groups. The dimer and the trimer of oligostyrene containing double bonds in the main chain were also examined as a reference.

\section{EXPERIMENTAL}

\section{Sample Preparation}

Three kinds of styrene oligomers were used in this experiment. The chemical structures of the samples are as follows: 
(I)<smiles>CCCCCCCCCCCCCCCCCCC</smiles>

(II)

$$
\begin{gathered}
\mathrm{CH}_{3}\left(\mathrm{CH}_{2}\right)_{3}\left(\underset{1}{\mathrm{CH}} \cdot \mathrm{CH}_{2}\right)_{n} \mathrm{H} \\
\mathrm{C}_{6} \mathrm{H}_{5}
\end{gathered}
$$

$$
\begin{aligned}
\mathrm{H}_{2} \mathrm{C}= & \underset{1}{\mathrm{C}}-\left(\mathrm{CH}_{2} \mathrm{CH}\right)_{n-1}-\mathrm{H} \\
& \stackrel{\mathrm{C}_{6} \mathrm{H}_{5}}{\mathrm{C}_{6} \mathrm{H}_{5}}
\end{aligned}
$$

Sample I has butyl groups at both ends, sample II has a butyl group at one end and a proton at the other, and sample III has protons at both ends. Samples I and II were prepared by ionic polymerization according to the reported procedure. ${ }^{11,12}$ The as-polymerized samples were obtained as mixtures of dimer to 20 -mer. Several samples having different number-average molecular weights were prepared by controlling the synthesis conditions.

Fractionation of the mixture of oligomers was carried out using a gel permeation chromatograph (GPC) of Nihon Bunseki Model LC-08 equipped with a $20 \mathrm{~mm}$ diameter column.

At the same time, several column systems which enabled separation of the sytrene oligomers were developed at our laboratory. The details of the fractionation were similar to those reported previously. ${ }^{13} \mathrm{~A}$ dimer and/or trimer rich fraction was also obtained by vacuum distillation, but it is separated into completely pure fractions, judging from the GPC diagram. Accordingly, the distilled samples were refractionated by GPC to obtain pure samples.

The structures of the dimer and the trimer were characterized by a high resolution NMR of Japan Electric Co.'s, JEOL-C60HL. Detailed characterization of samples I and II is found in previous reports. ${ }^{11,14}$

Samples II was prepared by Mr. Sekiguchi (Nihon University) who distilled thermally decomposed polystyrene. $^{15}$

\section{Measurements}

Differential Scanning Calorimetry (DSC). A differential scanning calorimeter (Perkin-Elmer DSC II) equipped with a low temperature environmental chamber was used. The environment of the sample holder was kept water-free and a stream of helium was made to flow through the apparatus during the heating and cooling runs. The sample weight was 3-
$5 \mathrm{mg}$ and the scanning rate was $5-20 \mathrm{~K} \mathrm{~min}^{-1}$.

Gel Permeation Chromatography (GPC). A gel permeation chromatography (Toyo Soda HLC801A) was used for analysis of the molecular weight. Each GPC peak was related to the number of main chain segments using a known dimer and trimer as the reference. The fractionation was carried out by a GPC of Nihon Bunseki (LC-08).

GPC curves were analyzed by a Curve Resolver (du Pont Model 310), assuming that each GPC peak was gaussian. The purity of the sample was calculated from the area of the resolved peaks.

Microscopy. A polarized light microscope (Leitz model ORTHOLUX) equipped with a heating stage and camera was used.

\section{RESULTS AND DISCUSSION}

Figure 1 represents the GPC chromatograms of the original samples I and II. The repeating number of the main chain segments, $n$, in each oligomer is indicated by a numerals in the figure. The chromatograms of the fractions obtained by vacuum distillation of the sample II are also shown in the figure. The dimer-rich fraction II-a contains the trimer, and the trimer-rich fraction II-b contains the dimer. These chromatograms show that a pure fraction was difficult to obtain by distillation alone. The sample became appreciably purer when the distilled fractions II-a and II-b were further separated by the

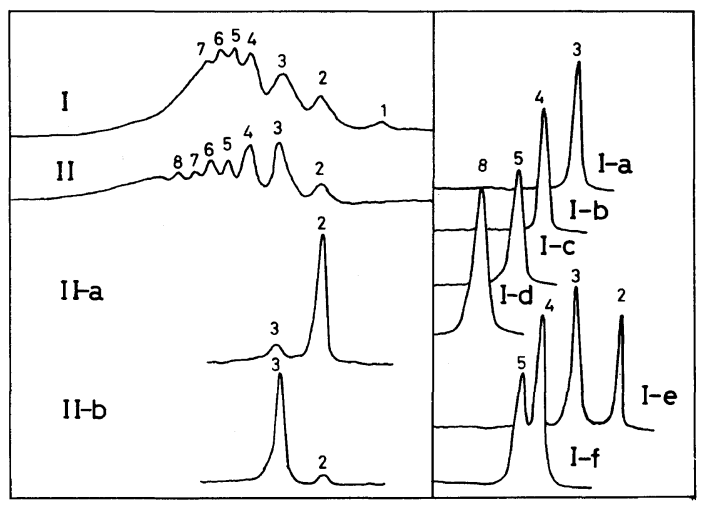

Elusion Volume

Figure 1. Gel permeation chromatograms of oligostyrenes: I, II, original samples I and II; II-a, II-b, distilled fractions of sample II; I-a, I-b, I-c, I-d, resolved fractions of sample I by GPC; I-e, I-f, mixtures of sample I. 
Glass Transition of Oligostyrene with Different End Groups

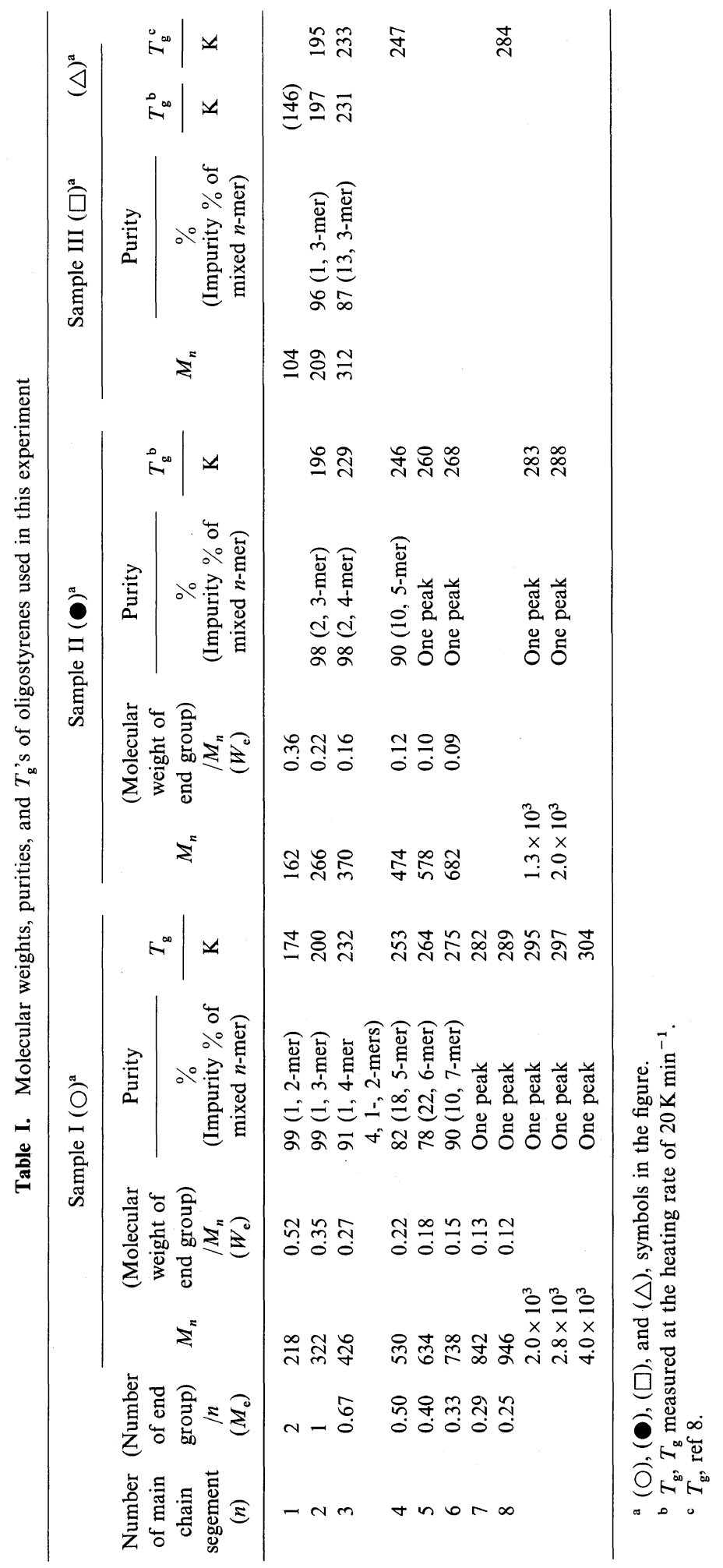


GPC. The right hand part of Figure 1 shows the fractions separated by GPC. The resolution of the two peaks becomes more and more difficult with increasing molecular weight since the resolution factor of the column decreases with increasing molecular weight. As shown in the chromatograms of the original samples, the peaks were distinguished up to 8-mer. Oligomers having a higher molecular weight than 8-mer were difficult to detect precisely by GPC. Accordingly, samples having a higher molecular weight than that 8-mer were evaluated from the number-average molecular weights, $M_{n}$ calculated from the elution number of GPC chromatograms.

The samples, the number of main chain segment, molecular weight and purity etc. are listed in Table I.

The monomer and dimer having butyl groups at both ends (sample I) crystallized gradually when the samples were kept in a refrigerator over a month. Flexible, long grass-like crystals of monomer were observed under a polarized light microscope (Photo. I). From the color of the polaized light, the molecular axis was found to be aligned along the longitudinal direction of the crystal. The peak temperature of the melting in DSC curve of the crystal was detected as the endotherm at $305 \mathrm{~K}$ at a heating rate of $10 \mathrm{~K} \mathrm{~min}^{-1}$, as shown in Figure 2.

The crystal of the dimer grown on the heating stage of the microscope at room temperature was plate-like. The peak temperature for the melting was detected at $319 \mathrm{~K}$ in the DSC curve, as can be seen from Figure 2. A small exothermic deviation observed before melting peak is attributed to premelt crystallization, and indicates that rearrangement of the imperfect part of the crystal takes place before melting. By using X-ray diffractometry, Morimoto ${ }^{16}$ found a periodical order of $17-18 \AA$ in the oligostyrene having an average-molecular weight of 600 . He suggested that the oligostyrene formed a smectic liquid crystal phase because the length $17-18 \AA$ corresponds to the chain length of the extended molecule. Other photographs (not shown here) as well as the presence of the pre-melt crystallization observed in the DSC curve suggest that the oligomer molecule was extended and arranged in a longitudinal direction. All the oligomer fractions and the styrene monomer became glassy when quenched from the molten state to temperatures lower than $T_{\mathrm{g}}$.

Typical DSC curves of oligostyrene fractions (sample I) taken at the heating rate of $20 \mathrm{~K} \mathrm{~min}^{-1}$ are shown in Figure 3. A typical dip of the base-line is clearly observed for these oligomer glasses. The $T_{\mathrm{g}}$ is defined as the point at which the extension of the base-line intersects the line tangent to the maximum slope of the endothermic deviation. The $T_{\mathrm{g}}$ 's thus evaluated from DSC curves are presented in Table I.

The heating rate dependence of the $T_{\mathrm{g}}$ of oligomers exhibited a trend similar to that of polystyrene. The temperature difference between the

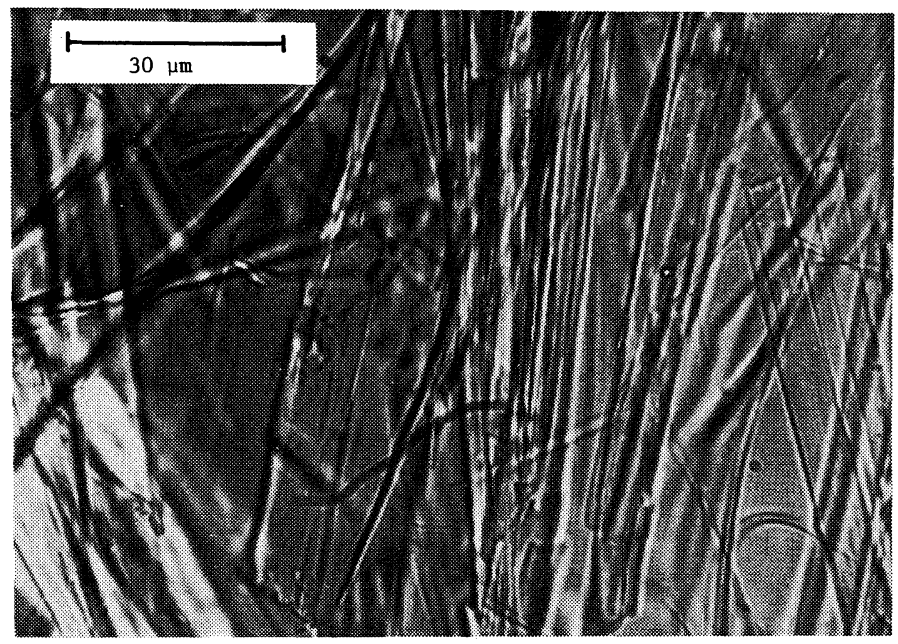

Photo I. Polarized light micrograph of styrene monomer having butyl grcups at both ends (sample I). Temperature, $290 \mathrm{~K}$. 


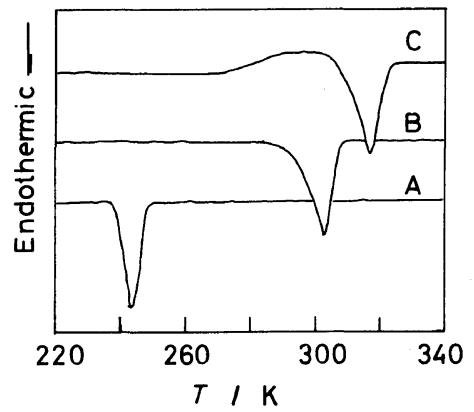

Figure 2. DSC curves of crystalline monomers and dimer: $\mathrm{A}, \mathrm{C}=\mathrm{C} ; \mathrm{B}, \mathrm{Bt}-\mathrm{C}-\mathrm{C}-\mathrm{Bt} ; \mathrm{C}, \mathrm{Bt}-(\mathrm{C}-\mathrm{C}-)_{2} \mathrm{Bt}$ (0)

Heating rate, $10 \mathrm{~K} \mathrm{~min}^{-1}$.

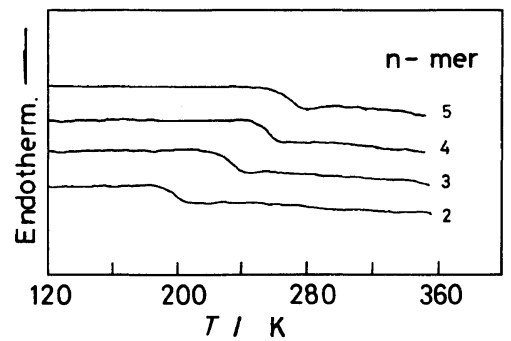

Figure 3. DSC curves of glassy oligostyrenes of sample I. Numerals indicate the number of main chain segments, $n$.

$T_{\mathrm{g}}$ 's measured at $20 \mathrm{~K} \mathrm{~min}^{-1}$ and those exptrapolated to the zero heating rate was about $6-8 \mathrm{~K}$ for the dimer to 8-mer.

The $T_{\mathrm{g}}$ 's of the three samples listed in Table I are plotted against the molecular weight, $M_{n}$, in Figure 4. The $T_{\mathrm{g}}$ 's increase almost linearly with $M_{n}$ up to about 600 and thereafter increase more slowly. Those for dimers and trimers do not fall on the same line, but the three lines converge for $M_{n}$ above 600.

Figure 5 shows $T_{\mathrm{g}}$ plotted against the reciprocal of $M_{n}$, where roman numerals show the samples and arabic numerals the number of main chain segments. The $T_{\mathrm{g}}$ 's of dimers having different end groups are almost identical, in spite of the large difference in molecular weight. The $T_{\mathrm{g}}$ 's of the dimers, for example, were observed within a temperarure range from $196-200 \mathrm{~K}$ while molecular weight ranged from 209-322. The $T_{\mathrm{g}}$ 's of trimer show a similar tendency, but the temperature range from $229-232 \mathrm{~K}$ and the molecular weights from

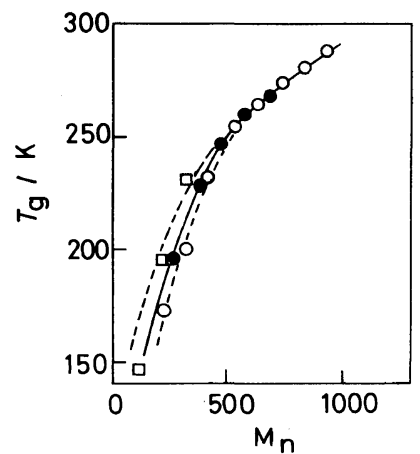

Figure 4. Relationship between $T_{\mathrm{g}}$ and $M_{n}: \bigcirc$, sample I;, , sample II; $\square$, sample III.

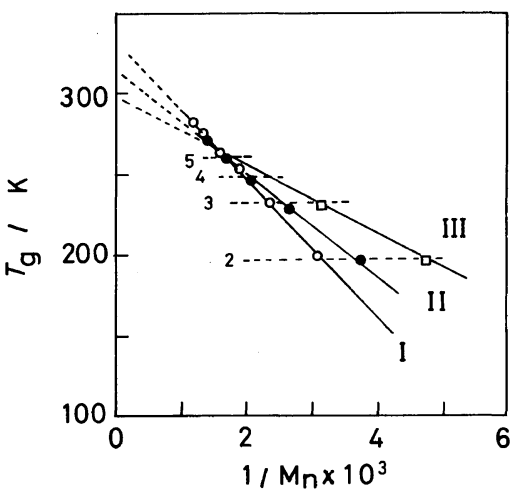

Figure 5. Relationship between $T_{\mathrm{g}}$ and $1 / M_{n}$ : I, sample I; II, sample II; III, sample III. Numerals indicate the number of main chain segments, $n$.

312-426. The three lines intersect at the $T_{\mathrm{g}}$ of $260 \mathrm{~K}$ which corresponds to the inflection point where the rate of increase in $T_{\mathrm{g}}$ changes in Figure 4. This $T_{\mathrm{g}}$ corresponds to a molecular weight of 600 .

Our data conform to the well-established relation between $T_{\mathrm{g}}$ and molecular weight:

$$
T_{\mathrm{g}}=A-B / M_{n}
$$

But $A$ and $B$ vary with the sample. Equation 1 for the samples I and II as are follows,

$$
\begin{aligned}
& T_{\mathrm{g}}=332-4.3 \times 10^{4} / M_{n} \\
& T_{\mathrm{g}}=316-3.2 \times 10^{4} / M_{n}
\end{aligned}
$$

If eq 1 is applied to the sample III whose data are insufficient for accurate determination of $A$ and $B$, $A=300$ and $B=2 \times 10^{4}$ can be obtained as tentative 


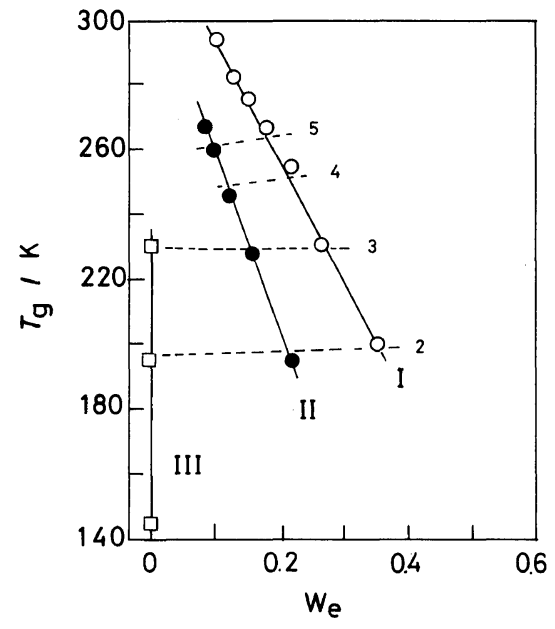

Figure 6. Relationship between $T_{\mathrm{g}}$ and $W_{\mathrm{e}}[=$ (molecular weight of end group) $/ M_{n}$ ]: I, sample I; II, sample II; III, sample III. Numerals indicate the number of main chain segments, $n$.

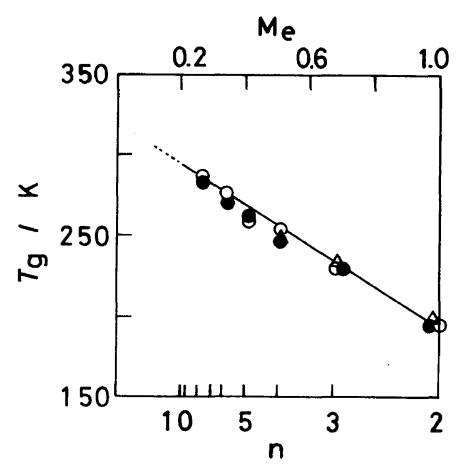

Figure 7. Relationship between $T_{\mathrm{g}}$ and $n$ (number of main chain segments) and $M_{\mathrm{e}}$ [=(number of end group)/ $n$ ]. Symbols are shown in Table I.

values. The constants $A$ and $B$ for samples I, II, and III vary and $B$ is high compared with that calculated by Boyer. ${ }^{17}$

The fact that $T_{\mathrm{g}}$ of $n$-mer does not change despite the large difference in the molecular weight can be seen clearly in Figure 6, where $T_{\mathrm{g}}$ is plotted against the molecular weight fraction of the end groups, $W_{\mathrm{e}}$, to the total molecular weight. The $T_{\mathrm{g}}$ 's obtained for different samples are found on separate lines, while the number of segments, $n$, are on similar $T_{\mathrm{g}}$ lines. The values of $T_{\mathrm{g}}$ for the samples I and II exptrapolated to $W_{\mathrm{e}}=0$ is in agreement with the temperature at which a discontinuity was found in the plot of $T_{\mathrm{g}}$ against the number of carbon atoms in the chain. ${ }^{10}$ It is clear from Figures 4, 5, and 6, that the $T_{\mathrm{g}}$ 's of $n$-mers are identical despite the difference in molecular weight, especially in the low molecular weight region.

The results presented in Figures 5 and 6 allow us to conclude that the $T_{\mathrm{g}}$ values plotted against the number of chain segments, $n$, should fall on a straight line. In fact, Uebbereiter and $\mathrm{Kanig}^{8}$ have already found the same for bulk-polymerized samples, although the end groups were not characterized. All reported values, ${ }^{7,8}$ together with those reported here are plotted in Figure 7. The value of $M_{\mathrm{e}}[=$ (number of end groups) $/ n]$ is also plotted on the abscissa. All the data points are well fitted by a single curve. Uebbereiter and Kanig consider that the end group function as a self-plasticizer in styrene oligomers.

If we replace the $M_{n}$ in eq 1 with the number of main chain segments, we can expect to obtain general constants. We modified eq 1 to eq 4 for comparison with the reported data. ${ }^{10}$

$$
1 / T_{\mathrm{g}}=A^{\prime}+B^{\prime} / n
$$

The calculated values of $A^{\prime}=2.90 \times 10^{-3}$ and $B^{\prime}=4.16 \times 10^{-3}$ accord reasonably well with those obtained previously. ${ }^{10}$

Summing up the results shown in Figures 6 and 7, it can be said that $T_{\mathrm{g}}$ 's of oligostyrenes are governed by the number of main chain segments rather than the size of end groups. In other words, a linear relationship independent of the end groups holds between the number of chain segments and $T_{\mathrm{g}}$ in the case of oligostyrenes.

If only the surface of phenomenon were considered, the above results might contradict themselves. However, if the self-plasticizing effect of the end group mentioned by Uebbereiter and Kanig is interpreted as a local relaxation of the end group, i.e., if the local movement of end groups already start at a temperature lower than $T_{\mathrm{g}}$, the end group motion cannot be the factor affecting the main chain motion near the glass transition temperature. For explaining what occurs in the molecules of styrene oligomers, Photo. I gives the idea that molecules carrying the same number of phenyl groups should array and aggregate together despite the motion of large end groups. Hence, the ordering of the main chain or the stacking of the phenyl group may be most important in considering the main chain motion of oligostyrene. 
Glass Transition of Oligostyrene with Different End Groups

\section{REFERENCES}

1. T. G Fox and P. J. Flory, J. Polym. Sci., 14, 315 (1954).

2. R. B. Beevers and E. G. T. White, Trans. Faraday Soc., 56, 744 (1960).

3. R. B. Beevers, J. Polym. Sci., A, 2, 5257 (1964).

4. E. V. Thompson, J. Polym. Sci., A-2, 4, 199 (1966).

5. J. M. G. Cowie and P. J. Toprowski, Eur. Polym. J., 4, 621 (1968).

6. J. M. G. Cowie and S. J. Bywater, J. Polym. Sci., C, 30, 85 (1970).

7. J. M. G. Cowie, Eur. Polym. J., 9, 1041 (1973).

8. K. Uebbereiter and G. J. Kanig, J. Colloid Sci., 7, 569 (1952).
9. K. Uebbereiter and U. Rohde-Liebenau, Macromol. Chem., 48, 164 (1961).

10. J. M. G. Cowie, Eur. Polym. J., 11, 297 (1975).

11. M. Iguchi, R. J. Ranson, and M. Gordon, Br. Polym. J., 5, 33 (1973).

12. T. Altares, D. P. Wyman, and V. R. Allen, J. Polym. Sci., A, 2, 4533 (1964).

13. H. Hatakeyama and T. Hatakeyama, Mokuzaigakkaishi, 23, 228 (1977).

14. S. Fujishige and N. Ohguri, Macromol. Chem., 176, 233 (1975).

15. T. Kuroki, T. Ogawa, Y. Sekiguchi, K. Saito, and T. Ikemura, Nihon Kagaku Zasshi, 11, 1717 (1977).

16. S. Morimoto, Nihon Kagaku Zasshi, 91, 182 (1970).

17. R. Boyer, Macromolecules, 7, 142 (1974). 